\title{
Acupuncture Treatment Compensated the Altered Functional Connectivity in the Pain Modulation System on Primary Dysmenorrhea: A Pilot Study
}

\author{
Cheng-Hao Tu ( $\sim$ lordowentu@gmail.com ) \\ China Medical University https://orcid.org/0000-0002-7695-5570 \\ Yu-Chen Lee \\ China Medical University Hospital Department of Acupuncture \\ Ying-Yu Chen \\ China Medical University Hospital Department of Chinese Medicine Gynecology \\ Chun-Ming Chen \\ China Medical University Hospital Department of Medical Imaging \\ Wen-Chi Lu \\ China Medical University Graduate Institute of Acupuncture Science \\ Yi-Hung Chen \\ China Medical University Graduate Institute of Acupuncture Science \\ Su-Tso Yang \\ China Medical University Hospital Department of Medical Imaging
}

\section{Research}

Keywords: primary dysmenorrhea, acupuncture, Sanyinjiao, descending pain modulation system, periaqueductal gray matter, functional connectivity, resting-state functional magnetic resonance imaging

Posted Date: November 12th, 2020

DOI: https://doi.org/10.21203/rs.3.rs-104133/v1

License: () (i) This work is licensed under a Creative Commons Attribution 4.0 International License. Read Full License 


\section{Abstract}

\section{Background}

Primary dysmenorrhea (PDM) is the most commonly encountered gynecological problem in reproductive-age women. We previously reported altered functional connectivity between periaqueductal gray matter and other pain-related brain regions in women with PDM, indicating a maladaptation in the descending pain modulation system. Clinically, acupuncture has been suggested as an effective treatment of PDM. Previous animal studies revealed that acupuncture on specific acupoint can produced analgesic effect via descending pain modulation system. In the present study, we used resting-state functional magnetic resonance imaging to investigate possible changes in descending pain modulation after acupuncture treatment in women with PDM.

Methods

Thirty-four right-handed adult women with PDM participated in this randomized, single-blinded, sham-controlled study. Each patient was randomly allocated to an 8-week verum or sham acupuncture intervention on the bilateral acupoint Sanyinjiao (SP6). Resting-state functional magnetic resonance imaging was conducted before, during, and after the intervention session to measure the spontaneous activity in brain. The functional connectivity maps between periaqueductal gray matter and other pain-related brain regions were generated to reveal the status of descending pain modulation systems.

Results

After 8 weeks, both groups reported decreased menstrual pain. However, the patterns of functional connectivity changes in descending pain modulation system were different after the verum or sham intervention. The effect of the verum acupuncture intervention may be underpinned by compensatory and/or resilience changes in descending pain modulation systems, whereas that of the sham acupuncture intervention may be underpinned by cognitive reappraisal of pain

Conclusions

The dissimilar pattern of the FC alterations after the verum and sham acupuncture indicating that different brain mechanisms may be responsible for the menstrual pain relief in verum or sham acupuncture intervention. These results may contribute to the discussion of whether acupuncture treatment induces therapeutic physiological alterations or its benefit is attributable to the placebo effect.

\section{Background}

Primary dysmenorrhea (PDM) is the most common gynecological problem among reproductive-age women. Around $10-20 \%$ of women experience PDM, causing them to be absent from work or school; thus, PDM affects their daily life (1). Women with PDM experience cramping pain during menses, which may be due to an abnormal concentration of prostanoids, uterine hypercontractility, or reduced uterine blood flow (2). Furthermore, they have a lower pain threshold in not only the referred pain site but also the non-referred pain site, indicating altered pain perception in the central nervous system $(3,4)$. We previously reported several abnormal functional and structural changes in brain regions associated with visceral sensation and pain processing $(5,6)$, as well as how altered functional connectivity (FC) between periaqueductal gray matter (PAG) and other pain-related brain regions in women with PDM indicated the maladaptation of the descending pain modulation system (7).

Acupuncture has been suggested to be an effective treatment for PDM (8). A recent meta-analysis including 60 randomized control trials reported that manual acupuncture is more effective for menstrual pain relief than no treatment and nonsteroidal antiinflammatory drugs (9). Among these 60 trials, the acupoint Sanyinjiao (SP6) was the most frequently used for treating PDM (9). Acupuncture might alleviate PDM through peripheral or central mechanisms. Peripherally, manual acupuncture on SP6 significantly improves uterine artery blood flow (10). However, blood prostanoid concentrations were not significantly different in response to electroacupuncture treatment on SP6 and that on other acupoints (11). Thus, the treatment effect of acupuncture on PDM may be mediated by vascular response but not changes in blood prostanoid levels. 
How acupuncture exerts the central effect on PDM has not been fully explored. Acupuncture on specific acupoints can produce an analgesic effect through descending pain modulation systems, especially by modulating PAG activity (12). Furthermore, the termination of chronic pain or of repetitive painful stimuli may be associated with functional and structural resilience changes in the brain (13-15). Therefore, we explored the central mechanisms possibly underlying the effect of acupuncture treatment on PDM. We hypothesized that acupuncture resilience the altered FC in the descending pain modulation systems between PAG and other pain-related brain areas on PDM.

\section{Materials And Methods}

\section{Subjects}

This study was conducted in accordance with the Declaration of Helsinki. The protocol was approved by the Institutional Review Board of China Medical University Hospital, Taiwan (CMUH105-REC1-027). All patients were recruited from the internet and then referred to and diagnosed by the Department of Chinese Medicine Gynecology, China Medical University Hospital, Taiwan. All patients received a full explanation of the study and signed written informed consent forms.

The inclusion criteria for subject were as follows: a) aged 20-35 years, b) right-handed, c) regular menstrual cycle of 27-32 days, and d) average menstrual pain level in the past 6 months $>4$ points on a 10-point numerical rating scale. The exclusion criteria were as follows: a) history of pituitary gland disorder; b) history of organic pelvic or reproductive system disease(s); c) history of mental disorder(s), such as major depressive disorder, bipolar disorder, general anxiety disorder, claustrophobia, or schizophrenia; d) history of brain trauma or neurological disease(s), such as epilepsy, stroke, Parkinson's disease, or dementia; e) history of or current pregnancy or plans to get pregnant; $\mathrm{f}$ ) presence of metal implants or a pacemaker; $\mathrm{g}$ ) use of oral contraceptives, psychiatric medicines, Chinese herbal medicine, or acupuncture treatment in the past 6 months or taking analgesic drugs $24 \mathrm{~h}$ before the magnetic resonance imaging (MRI) scan; and $\mathrm{h}$ ) unsuited for acupuncture treatment, as determined by the physicians of the Department of Chinese Medicine Acupuncture, China Medical University Hospital, Taiwan.

\section{Study design}

This study was a randomized, single-blind, sham-controlled study. All subjects were randomly allocated to the verum or sham acupuncture group at a 1:1 ratio by using a computer-generated list of random numbers. All subjects were not been inform the allocated group until the end of the experiment. During the inception interview, subject's general PDM experience in the past was assessed, and handedness were assessed using the Chinese version of the Edinburgh Handedness Inventory (16).

Transabdominal ultrasound was performed to exclude possible organic diseases in the reproduction system that may lead to secondary dysmenorrhea. The resting-state functional MRI (rfMRI) scans were conducted on weeks 0 , 4, and 8 of verum or sham acupuncture intervention sessions, respectively. To reduce the possible interference of menstrual phase, the MRI scans were conducted during the follicular phase (days 5-12 of the menstrual cycle). The latest menstrual pain experience and psychological state were also assessed after each MRI scan. Venous blood samples were taken to evaluate the concentrations of estrogen, progesterone, and testosterone within 2 days before or after MRI scans. If the subject had pregnancy concerns, a urine test strip pregnancy test was performed.

\section{Acupuncture intervention}

All acupuncture interventions were performed by attending physicians with more than 10-year experience in the outpatient clinics of the Department of Chinese Medicine Acupuncture, China Medical University Hospital, Taiwan. Subjects received a 20-min manual acupuncture intervention on bilateral SP6 twice per week for 8 consecutive weeks (total 16 interventions). The acupoint selection was based on a recent meta-analysis study which reported that manual acupuncture is more effective for menstrual pain relief and SP6 was the most frequently used for treating PDM (9). The acupoint was cleaned with an alcohol swab, and an 0ring was placed on the acupoint and covered by white surgical tape to conceal the penetration depth of the needle. In the verum acupuncture group, the standard disposable sterile acupuncture needle (diameter: $0.22 \mathrm{~mm}$, length: $30 \mathrm{~mm}$ ) was used to penetrate vertically into SP6 to a depth of approximately $25 \mathrm{~mm}$. In the sham acupuncture group, the Streitberger's placebo needle (Asiamed $\mathrm{GmbH}$. Pullach, Germany), which can effectively serve as the placebo needle in acupuncture $(17,18)$, was used to avoid skin penetration. In both verum and sham group, the retention time of needles on SP6 was 20 minutes and no manipulation on the 
needles during the retention time. To verify the effectiveness of single blinding procedure, the subjects were asked whether they received verum or sham acupuncture after they completed whole 8-week intervention.

\section{Menstrual pain experience and psychological assessments}

The Chinese version of the McGill Pain Questionnaire (MPQ) was used to assess the multidimensional menstrual pain experience (19). The total score of the pain rating index represents the menstrual pain experience, whereas that of the present pain intensity represents menstrual pain intensity. In addition, the Chinese version of Spielberger's State-Trait Anxiety Inventory (STAI) and the Chinese version of Beck's Depression Inventory II (BDI II) were used to assess the level of anxiety and depression, respectively (20, 21).

\section{Measurement of blood gonadal hormone level}

Blood concentrations of estrogen, progesterone, and testosterone were assessed using the chemiluminescent immunoassay sandwich method in the Department of Laboratory Medicine, China Medical University Hospital, Taiwan.

\section{Image acquisition}

All brain images were acquired with an 8-channel head coil in a 3.0 T MRI scanner (Signa HDxt, GE Healthcare, Chicago, IL, USA). For rfMRI, four blank scans and 200 resting scans were continuously acquired with an ascending interleaved echo-planar imaging sequence (repetition time $=2500 \mathrm{~ms}$; echo time $=30 \mathrm{~ms}$; flip angle $=90^{\circ}$; matrix $=64 \times 64$; field of view $=224 \times 224 \mathrm{~mm}^{2}$; slice number $=40$; and slice thickness $=3.5 \mathrm{~mm}$ ). High-resolution, three-dimensional, T1-weighted anatomical images were also acquired with a spoiled gradient echo sequence (repetition time: $7.356 \mathrm{~ms}$; echo time: $2.736 \mathrm{~ms}$; flip angle $=12^{\circ} ;$ matrix $=224 \times$ $224 \times 170$; field of view $=224 \times 224 \times 170 \mathrm{~mm}^{3}$ ). All scans were acquired in a dimly lit shielding room. Before scanning, subjects were instructed to relax, not to move their head, and keep their eyes opened during the scan. The whole time for scanning procedure (including preparation time) was about 20 minutes.

\section{Image preprocessing}

After discarding blank scans, the rfMRI scans were preprocessed using Statistical Parametric Mapping 12 (Wellcome Centre for Human Neuroimaging, University College London, London, UK) with MATLAB 2018a (MathWorks, Sherborn, MA, USA). The rfMRI images were first corrected for slice acquisition times and then realigned to correct the head motions during image scan. Subject with head motion above $1.75 \mathrm{~mm}$ maximum displacement in $\mathrm{x}$-, $\mathrm{y}$, or $\mathrm{z}$-direction or 1.5 degrees of angular motion were excluded for further analysis. The T1-weighted anatomical image was coregistered to the mean image generated from the realignment step and then normalized into the Montreal Neurological Institute reference space with default template using SPM12. The normalization parameters were then applied to all rfMRI images for spatial normalization with the resampled voxel size of $2 \times 2 \times$ $2 \mathrm{~mm}^{3}$. Finally, the normalized rfMRI images were smoothed with a three-dimensional Gaussian kernel (8-mm full width at half maximum).

The FC maps of PAG and other brain regions were generated by Data Processing and Analysis for Brain Imaging 4.3 with MATLAB 2018a (22). In preprocessed images, the brain activities in each voxel were linearly detrended and bandpass filtered (0.01$0.08 \mathrm{~Hz}$ ), and the confounding variables, namely six head movement parameters, the global mean signal, the mean signal of white matter, and the mean signal of the cerebrospinal fluid, were regressed out. The mean time-series activity in the seed region of PAG (a spherical region centered at the coordinates $[-4,-26,-14]$ with a 3-mm radius (23)) was extracted, and voxel-wise correlation analysis was conducted to generate the correlation maps of PAG. Finally, z-standardization was performed to generate standardized FC maps for statistical analysis.

\section{Statistical analysis}

The statistical analyses regarding demographic data, menstrual pain experience, psychological assessment, and blood gonadal hormone levels were conducted using SPSS v21 (IBM, Armonk, NY, USA). To compare intergroup differences in demographic data and general PDM experience, a two-sample $t$ test was conducted. The two-way mixed-model analysis of variance was used to test the possible interaction effects between groups (verum and sham) and times (before, during, and after) on menstrual pain experience, psychological status, and blood gonadal hormone levels. The significance was set at $p<0.05$. 
The statistical analysis for standardized FC maps of PAG was conducted using Statistical Parametric Mapping 12. The two-way mixed-model analysis of variance was constructed as the statistical model to estimate overall variance. The intragroup comparisons between different times in each group and interaction effect between groups and times were tested using $t$-contrasts in the model. A correlation analysis was also conducted between the menstrual pain experience and FC maps of PAG across 8weeks intervention in each group using two-way mixed-model analysis of covariance. Considering that the fMRI study often have very low statistical power (24), a less stringent significance threshold (uncorrected $p<0.005$ at the voxel level with a cluster size $>$ 40 , corresponding to an uncorrected cluster-level $p<0.35$ ) was applied to balance the chance of Type I and Type II error as suggested by a previous study (24).

\section{Results}

\section{Demographic data and general PDM experience}

Forty-six subjects were recruited, but 11 were excluded because of abnormal ultrasonography findings, taking hormone medicine, having a chronic pain history, and withdrawal of consent. Among the 35 subjects included, 19 were allocated to the verum acupuncture group and 16 to the sham acupuncture group. During the intervention session, one subject in the verum group withdrew her consent, leaving 18 patients in the verum group and 16 patients in the sham group who completed the intervention (Fig. 1).

Demographic data were not significantly different between the verum and sham groups in terms of age, handedness, age of menarche, gynecologic age, and length of menstrual cycle. No significant intergroup difference was noted in the age of PDM onset, duration of PDM history, duration of menstrual pain per cycle, menstrual pain experience, and menstrual pain intensity (Table 1). All subjects reported that they believed they were receiving the verum acupuncture intervention.

Table 1

Clinical and demographic data of women with primary dysmenorrhea

\begin{tabular}{|lccc|}
\hline & Verum (N=18) & Sham (N=16) & p-Value \\
\hline Age (year) & $24.89 \pm 4.59$ & $26.13 \pm 4.54$ & 0.44 \\
\hline Headedness (-1 1) & $0.84 \pm 0.16$ & $0.91 \pm 0.17$ & 0.23 \\
\hline The age of menarche (year) & $11.72 \pm 1.36$ & $11.88 \pm 1.36$ & 0.75 \\
\hline Gynecologic age (year) & $13.17 \pm 4.78$ & $14.25 \pm 4.81$ & 0.52 \\
\hline Length of menstrual cycle (day) & $31.06 \pm 2.34$ & $30.06 \pm 1.95$ & 0.19 \\
\hline The age of dysmenorrhea onset (year) & $14.50 \pm 2.73$ & $14.69 \pm 2.77$ & 0.84 \\
\hline Dysmenorrhea history (year) & $10.39 \pm 5.80$ & $11.44 \pm 5.46$ & 0.59 \\
\hline The duration of menstrual pain per cycle (day) & $1.89 \pm 0.68$ & $1.63 \pm 0.62$ & 0.25 \\
\hline MPQ & & & 0.91 \\
\hline PRI (0 78) & $35.83 \pm 13.28$ & $36.31 \pm 12.14$ & 0.83 \\
\hline PPI (0 5) & $3.17 \pm 1.20$ & $3.25 \pm 1.00$ & \\
\hline MPQ: McGill Pain Questionnaire; PRI: Pain Rating Index; PPI: Present Pain Intensity. All values are presented as mean \pm SD. \\
\hline
\end{tabular}

No significant interaction effect was found on menstrual pain experience or intensity between group and time factors. The main effect analysis revealed no effect on the group factor but a significant effect on the time factor. The post hoc analysis (controlled with Sidak correction) revealed that both menstrual pain experience and intensity significantly declined in the early stage of the 
intervention (weeks 0 to 4 ) and during the entire intervention (weeks 0 to 8 ), but no significant change was found in the late stage (weeks 4 to 8 ). Furthermore, no significant difference was observed in psychological status and gonadal hormone level (Table 2).

Table 2

Menstrual pain experience, psychological assessment, and gonadal hormone level across acupuncture intervention sessions

\begin{tabular}{|c|c|c|c|c|c|c|c|c|c|}
\hline & \multicolumn{3}{|c|}{ Verum $(\mathbf{N}=18)$} & \multicolumn{3}{|c|}{$\operatorname{Sham}(\mathrm{N}=16)$} & \multicolumn{3}{|l|}{ p-Value } \\
\hline & Week 0 & Week 4 & Week 8 & Week 0 & Week 4 & Week 8 & Interaction & Group & Time \\
\hline \multicolumn{10}{|l|}{ MPQ } \\
\hline $\begin{array}{l}\text { PRI total }(0 \sim \\
78)\end{array}$ & $\begin{array}{l}33.61 \\
\pm 13.22\end{array}$ & $\begin{array}{l}23.17 \pm \\
12.20\end{array}$ & $\begin{array}{l}26.11 \pm \\
13.79\end{array}$ & $\begin{array}{l}34.31 \\
\pm 14.78\end{array}$ & $\begin{array}{l}26.69 \\
\pm 15.70\end{array}$ & $\begin{array}{l}23.81 \pm \\
19.46\end{array}$ & 0.52 & 0.88 & $0.001^{\# \&}$ \\
\hline $\mathrm{PPI}(0$ 5) & $\begin{array}{l}2.83 \pm \\
1.38\end{array}$ & $\begin{array}{l}2.00 \pm \\
0.97\end{array}$ & $\begin{array}{l}2.17 \pm \\
1.20\end{array}$ & $\begin{array}{l}2.75 \pm \\
0.93\end{array}$ & $\begin{array}{l}2.19 \pm \\
0.98\end{array}$ & $\begin{array}{l}2.00 \pm \\
1.37\end{array}$ & 0.77 & 0.94 & $0.009^{\# \&}$ \\
\hline BDI & $\begin{array}{l}8.11 \pm \\
6.33\end{array}$ & $\begin{array}{l}7.89 \pm \\
5.80\end{array}$ & $\begin{array}{l}7.72 \pm \\
9.41\end{array}$ & $\begin{array}{l}10.69 \\
\pm 9.51\end{array}$ & $\begin{array}{l}6.38 \pm \\
3.65\end{array}$ & $\begin{array}{l}7.63 \pm \\
5.77\end{array}$ & 0.32 & 0.86 & 0.23 \\
\hline \multicolumn{10}{|l|}{ STAI } \\
\hline $\begin{array}{l}\text { State }(20 \sim \\
80)\end{array}$ & $\begin{array}{l}43.17 \\
\pm 8.96\end{array}$ & $\begin{array}{l}41.67 \pm \\
7.30\end{array}$ & $\begin{array}{l}40.78 \pm \\
10.94\end{array}$ & $\begin{array}{l}43.38 \\
\pm 9.98\end{array}$ & $\begin{array}{l}43.13 \\
\pm 8.88\end{array}$ & $\begin{array}{l}40.81 \pm \\
9.30\end{array}$ & 0.89 & 0.83 & 0.29 \\
\hline $\begin{array}{l}\text { Trait }(20 \sim \\
80)\end{array}$ & $\begin{array}{l}47.33 \\
\pm 7.22\end{array}$ & $\begin{array}{l}45.61 \pm \\
4.05\end{array}$ & $\begin{array}{l}45.72 \pm \\
10.01\end{array}$ & $\begin{array}{l}46.94 \\
\pm 9.77\end{array}$ & $\begin{array}{l}45.06 \\
\pm 8.95\end{array}$ & $\begin{array}{l}43.56 \pm \\
9.63\end{array}$ & 0.76 & 0.68 & 0.15 \\
\hline \multicolumn{10}{|l|}{ Hormones } \\
\hline $\begin{array}{l}\text { Estradiol } \\
(\mathrm{pg} / \mathrm{ml})\end{array}$ & $\begin{array}{l}87.89 \\
\pm 73.90\end{array}$ & $\begin{array}{l}63.44 \pm \\
30.63\end{array}$ & $\begin{array}{l}116.28 \pm \\
108.75\end{array}$ & $\begin{array}{l}77.69 \\
\pm 57.44\end{array}$ & $\begin{array}{l}94.63 \\
\pm 63.10\end{array}$ & $\begin{array}{l}117.44 \\
\pm 81.97\end{array}$ & 0.45 & 0.65 & 0.05 \\
\hline $\begin{array}{l}\text { Progesterone } \\
(\mathrm{ng} / \mathrm{ml})\end{array}$ & $\begin{array}{l}2.77 \pm \\
4.47\end{array}$ & $\begin{array}{l}0.75 \pm \\
1.29\end{array}$ & $\begin{array}{l}2.67 \pm \\
5.11\end{array}$ & $\begin{array}{l}1.23 \pm \\
2.61\end{array}$ & $\begin{array}{l}2.26 \pm \\
4.70\end{array}$ & $\begin{array}{l}2.77 \pm \\
4.12\end{array}$ & 0.24 & 0.98 & 0.40 \\
\hline $\begin{array}{l}\text { Testosterone } \\
(\mathrm{ng} / \mathrm{ml})\end{array}$ & $\begin{array}{l}0.52 \pm \\
0.28\end{array}$ & $\begin{array}{l}0.58 \pm \\
0.28\end{array}$ & $\begin{array}{l}0.64 \pm \\
0.27\end{array}$ & $\begin{array}{l}0.51 \pm \\
0.15\end{array}$ & $\begin{array}{l}0.49 \pm \\
0.15\end{array}$ & $\begin{array}{l}0.51 \pm \\
0.41\end{array}$ & 0.05 & 0.32 & 0.05 \\
\hline
\end{tabular}

\section{Changes in FC maps across acupuncture sessions}

Due to scanner scheduling issue, three and four subjects in the verum and sham groups, respectively, were unable to undergo the rfMRI on week 4 . Thus, only 15 and 12 subjects, respectively, were included in the imaging analysis.

After the 8-week acupuncture intervention, in the verum group, increased FC was found in the right middle frontal gyrus, right inferior occipital gyrus, right cuneus, right parahippocampal gyrus, midbrain, and cerebellum, and decreased FC was evident in the right inferior frontal gyrus, right middle temporal gyrus, right anterior cingulate gyrus, left precentral gyrus, left inferior parietal lobule, and left angular gyrus (Fig. 2, Table 3). In the sham group, increased FC was found in the right superior parietal lobule, left temporal gyrus, midbrain, and cerebellum, whereas decreased FC was evident in the right rectal gyrus, right superior frontal gyrus, right inferior frontal gyrus, left inferior temporal gyrus, and bilateral inferior parietal lobule (Fig. 3, Table 3). After the 8-week intervention, the interaction analysis revealed that compared with the sham group, the verum group had a higher increase in FC in the right middle frontal gyrus, right caudate body, and cerebellum and a higher decrease in FC in the left precentral gyrus, bilateral precuneus, and brainstem (Table 3). 
Table 3

Changes in functional connectivity map after acupuncture intervention

Week $0>$ Week 8

Coordinate

Anatomical BA Size $Z \max \quad x \quad y \quad z$

Area

Verum

R Ant

Cingulate

$\begin{array}{llllll}32 & 112 & 3.86 & 18 & 24 & 42\end{array}$

$\mathrm{G}$

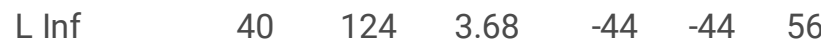

Parietal

Lob

Precentra

G

$\begin{array}{lllllll}\mathrm{L} \text { Angular } & 39 & 419 & 3.44 & -40 & -78 & 30\end{array}$

L

Paracentral

$\begin{array}{llllll}31 & 47 & 3.37 & -6 & -24 & 46\end{array}$

Lob

$\mathrm{R} \operatorname{lnf}$

Frontal G

$\begin{array}{llllll}4 / 6 & 347 & 3.56 & -24 & -30 & 70\end{array}$

R Cuneus

Bil Midbrain

Anatomical Area

BA

Coordinate

Week $0<$ Week 8

$\begin{array}{lllllll}\text { R Mid } & 39 & 87 & 3.24 & 42 & -60 & 24\end{array}$

Temporal

G

\begin{tabular}{|c|c|c|c|c|c|c|c|c|c|c|c|c|c|}
\hline & & & & & & & \multicolumn{2}{|l|}{$\begin{array}{l}\text { L Cerebellum } \\
\text { Culmen }\end{array}$} & 45 & 3.09 & -34 & -50 & -36 \\
\hline & & & & & & & \multicolumn{2}{|l|}{$\begin{array}{l}\text { L Cerebellum } \\
\text { Vermis }\end{array}$} & 79 & 3.08 & 0 & -78 & -20 \\
\hline & & & & & & & $\begin{array}{l}\text { R } \\
\text { Parahippocampal } \\
\text { G }\end{array}$ & 30 & 46 & 2.67 & 18 & -44 & 8 \\
\hline \multicolumn{14}{|l|}{ Sham } \\
\hline \multirow[t]{2}{*}{ R Rectal G } & 25 & 63 & 4.12 & 14 & 12 & -22 & \multicolumn{2}{|l|}{ L Midbrain } & 52 & 4.06 & -8 & -26 & -16 \\
\hline & 11 & 68 & 3.35 & 14 & 30 & -22 & \multicolumn{2}{|l|}{$\begin{array}{l}\text { L } \\
\text { Parahippocampal } \\
\text { G }\end{array}$} & 53 & 3.50 & -26 & -22 & -14 \\
\hline $\begin{array}{l}\text { R Sup } \\
\text { Frontal G }\end{array}$ & 8 & 70 & 3.45 & 10 & 26 & 54 & \multicolumn{2}{|l|}{$\begin{array}{l}\text { R Cerebellum } \\
\text { Vermis }\end{array}$} & 142 & 3.34 & 0 & -50 & -36 \\
\hline \multirow{2}{*}{$\begin{array}{l}\text { Bil Inf } \\
\text { Parietal } \\
\text { Lob }\end{array}$} & 40 & 147 & 3.30 & -52 & -48 & 36 & $\begin{array}{l}\text { R Sup Parietal } \\
\text { Lob }\end{array}$ & 7 & 60 & 3.29 & 34 & -82 & 44 \\
\hline & & 43 & 3.03 & 54 & -42 & 28 & L Mid Temporal G & 21 & 60 & 3.09 & -44 & -6 & -20 \\
\hline $\begin{array}{l}\text { L Inf } \\
\text { Temporal } \\
\text { G }\end{array}$ & 21 & 148 & 3.26 & -66 & -28 & -20 & \multicolumn{2}{|l|}{$\begin{array}{l}\text { L Cerebellar } \\
\text { Tonsil }\end{array}$} & 47 & 3.06 & -12 & -46 & -46 \\
\hline $\begin{array}{l}\mathrm{R} \text { Inf } \\
\text { Frontal G }\end{array}$ & 47 & 121 & 3.19 & 50 & 38 & 0 & & & & & & & \\
\hline
\end{tabular}




\begin{tabular}{|c|c|c|c|c|c|c|c|c|c|c|c|c|c|}
\hline \multicolumn{7}{|c|}{ Week $0>$ Week 8} & \multicolumn{7}{|l|}{ Week $0<$ Week 8} \\
\hline & 44 & 77 & 3.04 & 60 & 18 & 16 & & & & & & & \\
\hline \multicolumn{14}{|l|}{$\begin{array}{l}\text { Verum > } \\
\text { Sham }\end{array}$} \\
\hline $\begin{array}{l}\mathrm{R} \\
\text { Brainstem }\end{array}$ & & 43 & 3.67 & 20 & -22 & -50 & $\begin{array}{l}\text { Bil Cerebellum } \\
\text { Culmen }\end{array}$ & & 81 & 3.77 & -4 & -36 & -6 \\
\hline \multirow{2}{*}{$\begin{array}{l}\text { Bil } \\
\text { Precuneus }\end{array}$} & 19 & 112 & 3.64 & 34 & -82 & 44 & & & & 3.65 & 4 & -40 & -6 \\
\hline & 19 & 102 & 3.21 & -34 & -76 & 34 & R Mid Frontal G & $46 / 10$ & 322 & 3.52 & 44 & 48 & 10 \\
\hline $\begin{array}{l}\text { L } \\
\text { Precentral } \\
\text { G }\end{array}$ & $4 / 6$ & 168 & 3.33 & -24 & -30 & 68 & R Caudate Body & & 60 & 3.50 & 12 & -6 & 24 \\
\hline
\end{tabular}

BA: Brodmann area; Size: number of voxels in the cluster; Zmax: peak Z value; L: left; R: right; Bil: bilateral; Sup: superior; Inf: inferior; Mid: middle; Med: medial; Ant: anterior; G: gyrus; Lob: lobule; N: nucleus.

After the early stage of intervention, in the verum group, increased FC was evident in the left medial frontal gyrus, left superior temporal gyrus, left parahippocampal gyrus, and cerebellum, and decreased FC was evident in the right superior frontal gyrus, left precentral gyrus, left paracentral lobule, and bilateral precuneus. In the sham group, increased FC was identified in the right middle occipital gyrus, left precentral gyrus, and cerebellum, whereas decreased FC was apparent in the right medial frontal gyrus, right superior temporal gyrus, right middle temporal gyrus, and cerebellum. The interaction analysis revealed that compared with the sham group, the verum group had a higher increase in FC in the right middle temporal gyrus, left superior temporal gyrus, and cerebellum and a higher decrease in FC in the right middle occipital gyrus and left superior frontal gyrus (Table S1).

After the late stage of intervention, in the verum group, increased FC was apparent in the left middle frontal gyrus, left middle occipital gyrus, left precuneus, bilateral cuneus, and bilateral parahippocampal gyrus, whereas decreased FC was evident in the right inferior parietal lobule, left medial frontal gyrus, left supramarginal gyrus, and bilateral middle temporal gyrus. In the sham group, increased FC was identified in the right inferior parietal lobule, left precentral gyrus, left superior temporal gyrus, bilateral posterior cingulate gyrus, and cerebellum, and decreased FC was apparent in the right middle frontal gyrus, right inferior frontal gyrus, left inferior parietal lobule, left fusiform gyrus, bilateral superior frontal gyrus, and bilateral anterior cingulate gyrus. The interaction analysis revealed that compared with the sham group, the verum group had a higher increase in FC in the left paracentral lobule, left fusiform gyrus, left caudate body, and bilateral superior frontal gyrus and a higher decrease in FC in the right interior parietal lobule, left precentral gyrus, left middle temporal gyrus, and cerebellum (Table S2).

\section{Correlation between menstrual pain experience and the FC of PAG}

In the verum group, menstrual pain experience was positively correlated with the FC of PAG in the right middle frontal gyrus, right subcallosal gyrus, left precentral gyrus, and bilateral caudate head and negatively correlated with the right inferior temporal gyrus, right lingual gyrus, left postcentral gyrus, and left superior parietal lobule (Fig. 4, Table 4). In the sham group, menstrual pain experience was positively correlated with the FC of PAG in the right inferior frontal gyrus, left middle temporal gyrus, left fusiform gyrus, left cingulate gyrus, bilateral cuneus, and cerebellum and negatively correlated with the right inferior temporal gyrus, left caudate body, bilateral thalamus, and cerebellum (Fig. 4, Table 4). The interaction analysis revealed that compared with the sham group, the menstrual pain experience in the verum group was more positively correlated with the FC of PAG in the right middle frontal gyrus, right postcentral gyrus, right inferior temporal gyrus, right caudate head, left precentral gyrus, and left middle temporal gyrus and more negatively correlated in the left cuneus, left anterior cingulate gyrus, and cerebellum (Table 4). 
Table 4

Correlation between menstrual pain experience and functional connectivity of PAG

\begin{tabular}{|c|c|c|c|c|c|c|c|c|c|c|c|c|c|}
\hline \multicolumn{7}{|l|}{ Positive } & \multicolumn{7}{|l|}{ Negative } \\
\hline & & & & \multicolumn{7}{|c|}{ Coordinate } & \multicolumn{3}{|c|}{ Coordinate } \\
\hline $\begin{array}{l}\text { Anatomical } \\
\text { Area }\end{array}$ & BA & Size & $Z \max$ & $x$ & $y$ & z & Anatomical Area & BA & Size & Zmax & $x$ & y & z \\
\hline \multicolumn{14}{|l|}{ Verum } \\
\hline \multirow{2}{*}{$\begin{array}{l}\text { Bil Caudate } \\
\text { Head }\end{array}$} & & 463 & 3.92 & 12 & 18 & 6 & L Postcentral G & 2 & 59 & 4.01 & -30 & -42 & 72 \\
\hline & & 44 & 2.83 & -14 & 18 & 4 & R Inf Temporal G & 20 & 90 & 3.84 & 58 & -28 & -28 \\
\hline $\begin{array}{l}\text { R Subcallosal } \\
\text { G }\end{array}$ & 13 & 463 & 3.32 & 18 & 16 & -14 & $\begin{array}{l}\text { L Sup Parietal } \\
\text { Lob }\end{array}$ & 7 & 79 & 3.51 & -40 & -60 & 60 \\
\hline R Mid Frontal G & 6 & 137 & 3.28 & 34 & 6 & 54 & R Lingual G & 18 & 87 & 3.28 & 12 & -78 & -14 \\
\hline L Precentral G & 6 & 88 & 3.18 & -26 & -20 & 54 & & & & & & & \\
\hline \multicolumn{14}{|l|}{ Sham } \\
\hline \multirow[t]{2}{*}{ Bil Cuneus } & 19 & 927 & 4.65 & 32 & -86 & 20 & R Inf Temporal G & 20 & 89 & 4.47 & 36 & -8 & -44 \\
\hline & 19 & 635 & 3.68 & -16 & -98 & 20 & $\begin{array}{l}\text { R Cerebellum } \\
\text { Culmen }\end{array}$ & & 75 & 3.37 & 8 & -60 & -26 \\
\hline $\begin{array}{l}\text { L Mid } \\
\text { Temporal G }\end{array}$ & 21 & 62 & 3.26 & -54 & 8 & -16 & L Caudate Body & & 69 & 3.21 & -20 & -6 & 24 \\
\hline $\begin{array}{l}\text { L Ant Cingulate } \\
\text { G }\end{array}$ & 32 & 61 & 3.26 & -16 & 14 & 32 & Bil Thalamus & & 73 & 2.99 & -4 & -2 & 4 \\
\hline L Fusiform G & 37 & 74 & 3.20 & -38 & -62 & -16 & & & & 2.82 & 2 & -8 & 4 \\
\hline $\begin{array}{l}\text { L Cerebellum } \\
\text { Lob }\end{array}$ & & 48 & 3.08 & -4 & -68 & -52 & & & & & & & \\
\hline R Inf Frontal G & 47 & 43 & 3.06 & 28 & 12 & -22 & & & & & & & \\
\hline \multicolumn{14}{|l|}{ Verum > Sham } \\
\hline $\begin{array}{l}\text { R Inf Temporal } \\
\text { G }\end{array}$ & 20 & 64 & 3.85 & 36 & -6 & -40 & Bil Cuneus & 19 & 432 & 3.85 & -26 & -88 & 34 \\
\hline R Mid Frontal G & 6 & 212 & 3.51 & 34 & 6 & 56 & & 19 & 356 & 3.72 & 34 & -90 & 18 \\
\hline $\begin{array}{l}\text { R Caudate } \\
\text { Head }\end{array}$ & & 187 & 3.48 & 12 & 18 & 6 & $\begin{array}{l}\text { R Cerebellum } \\
\text { Tuber }\end{array}$ & & 76 & 3.68 & 30 & -90 & -38 \\
\hline L Precentral G & 6 & 87 & 3.37 & -26 & -20 & 56 & L Cerebellum Lob & & 91 & 3.21 & -26 & -74 & -58 \\
\hline $\begin{array}{l}\text { L Mid } \\
\text { Temporal G }\end{array}$ & 21 & 62 & 3.21 & -36 & 6 & -38 & L Cingulate G & 24 & 52 & 3.13 & -14 & 14 & 34 \\
\hline R Postcentral G & 3 & 46 & 3.00 & 22 & -30 & 62 & & & & & & & \\
\hline
\end{tabular}

BA: Brodmann area; Size: number of voxels in the cluster; Zmax: peak Z value; L: left; R: right; Bil: bilateral; Sup: superior; Mid: middle; Inf: inferior; Ant: anterior; G: gyrus; Lob: lobule.

\section{Discussion}

We conducted this rfMRI study to investigate the possible central mechanisms of acupuncture treatment on PDM in terms of FC changes within the descending pain modulation systems. Both verum and sham acupuncture significantly reduced menstrual 
pain experience and menstrual pain intensity after 8 weeks of intervention. However, in the verum group, the FC changes were mainly observed in the regions associated with affective and cognitive pain modulation and attention-related pain modulation, whereas in the sham group, the FC changes were mainly evident in the regions associated with cognitive reappraisal of pain. This implies that the relief of menstrual pain in the two groups may be caused by different brain mechanisms.

The FC changes after verum acupuncture may be associated with compensatory responses on PDM. We previously reported that at menstrual pain onset, compared with healthy controls, women with PDM showed a reactive increase in the FC of PAG in the sensorimotor cortex and a maladaptive decrease in the FC of PAG in the dorsolateral prefrontal cortex (dIPFC) and medial prefrontal cortex (mPFC) (7). We also reported an adaptive positive correlation to the affective dimension of menstrual pain in the ventrolateral prefrontal cortex (VIPFC) at menstrual pain onset. Moreover, adaptive hypoconnectivity in the posterior parietal cortex (PPC) and maladaptive hypoconnectivity in the posterior cingulate cortex (PCC) were found during the nonpainful periovulatory phase (7). In the present study, increased FC was observed in the vIPFC and decreased FC was evident in sensorimotor regions at menstrual pain offset after 8 weeks of verum acupuncture intervention. Because the vIPFC may be associated with the modulation of pain-related emotion (25), the increased FC in the vIPFC may reflect enhanced affective pain modulation to reduce menstrual pain. The decreased FC in the sensorimotor cortex may be associated with the tuning-down effect from acupuncture. A previous study reported lower degrees of FC in the sensorimotor cortex in patients with low-intensity chronic low back pain than in patients with high-intensity chronic low back pain (26). Acupuncture may immediately modulate the FC of sensorimotor regions to other brain regions (27). Since the FC in the sensorimotor region remained positively correlated with menstrual pain experience (reflecting the nature of menstrual pain experience), the decreased FC in the absence of menstrual pain may represent the tuningdown effect accumulated from repeated acupuncture treatments. In addition, an adaptive decrease in the FC in the PPC, a region associated with attention-related pain modulation (28), was still observed after verum acupuncture intervention. Taken together, our findings indicate that the relief of menstrual pain after 8 weeks of acupuncture intervention may be associated with compensatory responses in descending pain modulation networks having an effect on PDM.

The effect of acupuncture intervention on PDM may occur through such compensatory responses as well as resilience responses in descending pain modulation systems. After 4 weeks of acupuncture intervention, increased FC was evident in the mPFC, a region associated with pain appraisal and experience $(29,30)$. Since both menstrual pain experience and intensity decreased after 4 weeks, the increased FC in the MPFC may have been due to the resilience response of maladaptive changes in the mPFC on PDM (7). This notion is further supported by the observations that menstrual pain experience was slightly (but not significantly) increased in the late intervention stage, whereas the FC in the MPFC decreased. Moreover, the maladaptive change of decreased FC in the PCC was not observed after verum acupuncture intervention, indicating that further maladaptive changes in the PCC were prevented. Hence, the treatment effect of verum acupuncture may also be underpinned by the resilience responses in descending pain modulation systems.

FC changes after sham acupuncture interventions may be associated with cognitive reappraisal of pain. In the present study, decreased FC was observed in the vIPFC, orbitofrontal cortex (OFC), and PPC, and increased FC was evident in the parahippocampal gyrus after 8 weeks of sham acupuncture intervention. The vIPFC and OFC may play critical roles in the placebo effect on pain $(31,32)$. The vIPFC is activated when patients believe their pain can be controlled, whereas the OFC is involved in reevaluation of information $(33,34)$. The function of these two regions may contribute to the cognitive reappraisal of pain, which underlies the placebo effect (35). In addition, the PPC is also associated with the cognitive reappraisal of emotion (36). Combined with the belief that one is receiving verum acupuncture, the treatment effect of sham acupuncture intervention may be due to the cognitive reappraisal of menstrual pain in descending pain modulation systems but not to resilience to altered FC. This notion is further supported by the positive correlation between FC in the OFC and menstrual pain experience. Because PAG is most activated when a pain cue is present (32), desynchronization (i.e., decreased FC) between PAG and other brain regions may occur when a patient expects menstrual pain relief after sham acupuncture. Thus, lower FC may be associated with reduced menstrual pain experience.

Our study has several limitations. First, this was a pilot study, meaning that the number of subjects was small, which may lower the statistical power. Second, to balance the chance of type I and type II errors in fMRI study, we applied a less stringent significance threshold as suggested by previous literature, which may have yielded only suggestive results. Third, the MPQ is designed for general pain and not specifically for menstrual pain. Thus, future studies with a larger sample size and a specific

Page 10/22 
menstrual pain questionnaire may be needed to elucidate the brain mechanisms underlying the effect of acupuncture treatment on PDM.

\section{Conclusions}

In conclusion, our results demonstrated that both verum and sham acupuncture intervention on bilateral SP6 can significantly relieve menstrual pain. However, the two interventions exhibited different patterns of FC changes in altered descending pain modulation systems. The effect of the verum acupuncture intervention may be underpinned by compensatory and/or resilience changes, whereas that of the sham acupuncture intervention may be underpinned by cognitive reappraisal of pain. The results of this pilot study may be a basis for further discussion of whether acupuncture treatment induces physiological therapeutic alterations or simply induces the placebo effect.

\section{Abbreviations}

BDI II

Beck's Depression Inventory II

dIPFC

dorsolateral prefrontal cortex

FC

functional connectivity

mPFC

medial prefrontal cortex

MPQ

McGill Pain Questionnaire

MRI

magnetic resonance imaging

OFC

orbitofrontal cortex

PAG

periaqueductal gray matter

PCC

posterior cingulate cortex

PDM

primary dysmenorrhea;

PPC

posterior parietal cortex

rMRI

resting-state magnetic resonance imaging

STAI

State-Trait Anxiety Inventory

vIPFC

ventrolateral prefrontal cortex

\section{Declarations}

\section{Ethics approval and consent to participate}

This study was conducted in accordance with the Declaration of Helsinki. The protocol was approved by the Institutional Review Board of China Medical University Hospital, Taiwan (CMUH105-REC1-027). All patients received a full explanation of the study and signed written informed consent forms. 
Not applicable.

Availability of data and materials

All data analyzed during this study are available from the corresponding author on reasonable request.

\section{Competing interest}

The authors declare that they have no competing interests.

\section{Funding}

This work was supported by the Ministry of Science and Technology, Taiwan (MOST 106-2314-B-039-012, 107-2314-B-039-058MY2, and 108-2813-C-039-141-B) and the China Medical University, Taiwan (CMU 102-ASIA-19, 107-N-24, and 108-MF-03).

\section{Author's contributions}

Conceptualization, C.H.T. and S.T.Y.; Patient diagnosis, Y.Y.C.; Patient intervention, Y.C.L.; Data acquisition, C.M.C. and W.C.L; Formal analysis, C.H.T and W.C.L.; Writing-original draft preparation, C.H.T.; Writing-review and editing, C.H.T, S.T.Y, and Y.H.C. All authors have read and agree with the final manuscript.

\section{Acknowledgements}

The authors thank the English-editing service by Wallace Academic Editing, Taipei, Taiwan for this manuscript.

\section{References}

1. French L. Dysmenorrhea. Am Fam Physician. 2005;71(2):285-91.

2. Dawood MY. Primary dysmenorrhea: advances in pathogenesis and management. Obstet Gynecol. 2006;108(2):428-41.

3. Bajaj P, Madsen H, Arendt-Nielsen L. A comparison of modality-specific somatosensory changes during menstruation in dysmenorrheic and nondysmenorrheic women. Clin J Pain. 2002;18(3):180-90.

4. Granot M, Yarnitsky D, Itskovitz-Eldor J, Granovsky Y, Peer E, Zimmer EZ. Pain perception in women with dysmenorrhea. Obstet Gynecol. 2001;98(3):407-11.

5. Tu CH, Niddam DM, Chao HT, Chen LF, Chen YS, Wu YT, et al. Brain morphological changes associated with cyclic menstrual pain. Pain. 2010;150(3):462-8.

6. Tu CH, Niddam DM, Chao HT, Liu RS, Hwang RJ, Yeh TC, et al. Abnormal cerebral metabolism during menstrual pain in primary dysmenorrhea. Neuroimage. 2009;47(1):28-35.

7. Wei SY, Chao HT, Tu CH, Li WC, Low I, Chuang CY, et al. Changes in functional connectivity of pain modulatory systems in women with primary dysmenorrhea. Pain. 2016;157(1):92-102.

8. Organization WH. Acupuncture: Review and Analysis of Reports on Controlled Clinical Trials. Geneva: World Health Organization; 2003.

9. Woo HL, Ji HR, Pak YK, Lee H, Heo SJ, Lee JM, et al. The efficacy and safety of acupuncture in women with primary dysmenorrhea: A systematic review and meta-analysis. Medicine (Baltimore). 2018;97(23):e11007.

10. Yu YP, Ma LX, Ma YX, Ma YX, Liu YQ, Liu CZ, et al. Immediate effect of acupuncture at Sanyinjiao (SP6) and Xuanzhong (GB39) on uterine arterial blood flow in primary dysmenorrhea. J Altern Complement Med. 2010;16(10):1073-8.

11. Shi GX, Liu CZ, Zhu J, Guan LP, Wang DJ, Wu MM. Effects of acupuncture at Sanyinjiao (SP6) on prostaglandin levels in primary dysmenorrhea patients. Clin J Pain. 2011;27(3):258-61.

12. Lin JG, Chen WL. Acupuncture analgesia: a review of its mechanisms of actions. Am J Chin Med. 2008;36(4):635-45. 
13. Rodriguez-Raecke R, Niemeier A, Ihle K, Ruether W, May A. Brain gray matter decrease in chronic pain is the consequence and not the cause of pain. J Neurosci. 2009;29(44):13746-50.

14. Teutsch S, Herken W, Bingel U, Schoell E, May A. Changes in brain gray matter due to repetitive painful stimulation. Neuroimage. 2008;42(2):845-9.

15. Bingel U, Herken W, Teutsch S, May A. Habituation to painful stimulation involves the antinociceptive system-a 1-year followup of 10 participants. Pain. 2008;140(2):393-4.

16. Yang N, Waddington G, Adams R, Han J. Translation, cultural adaption, and test-retest reliability of Chinese versions of the Edinburgh Handedness Inventory and Waterloo Footedness Questionnaire. Laterality. 2018;23(3):255-73.

17. Xie CC, Wen XY, Jiang L, Xie MJ, Fu WB. Validity of the "streitberger" needle in a chinese population with acupuncture: a randomized, single-blinded, and crossover pilot study. Evid Based Complement Alternat Med. 2013;2013:251603.

18. Streitberger K, Kleinhenz J. Introducing a placebo needle into acupuncture research. Lancet. 1998;352(9125):364-5.

19. Hui YL, Chen AC. Analysis of headache in a Chinese patient population. Ma Zui Xue Za Zhi. 1989;27(1):13-8.

20. Wu PC, Huang TW. Gender-related invariance of the Beck Depression Inventory II for Taiwanese adolescent samples. Assessment. 2014;21(2):218-26.

21. Ma WF, Liu YC, Chen YF, Lane HY, Lai TJ, Huang LC. Evaluation of psychometric properties of the Chinese Mandarin version State-Trait Anxiety Inventory $Y$ form in Taiwanese outpatients with anxiety disorders. Journal of psychiatric and mental health nursing. 2013;20(6):499-507.

22. Yan CG, Wang XD, Zuo XN, Zang YF. DPABI: Data Processing \& Analysis for (Resting-State) Brain Imaging. Neuroinformatics. 2016;14(3):339-51.

23. Kong J, Tu PC, Zyloney C, Su TP. Intrinsic functional connectivity of the periaqueductal gray, a resting fMRI study. Behav Brain Res. 2010;211(2):215-9.

24. Cremers HR, Wager TD, Yarkoni T. The relation between statistical power and inference in fMRI. PLoS One. 2017;12(11):e0184923.

25. Garcia-Larrea L, Peyron R. Pain matrices and neuropathic pain matrices: a review. Pain. 2013;154 Suppl 1:S29-43.

26. Kong J, Spaeth RB, Wey HY, Cheetham A, Cook AH, Jensen K, et al. S1 is associated with chronic low back pain: a functional and structural MRI study. Mol Pain. 2013;9:43.

27. Bian Y, He X, Hu S, Li C, Xu C, Kan H, et al. Functional Connectivity Modulation by Acupuncture in Patients with Bell's Palsy. Evid Based Complement Alternat Med. 2016;2016:5928758.

28. Villemure C, Bushnell MC. Mood influences supraspinal pain processing separately from attention. J Neurosci. 2009;29(3):705-15.

29. Apkarian AV, Baliki MN, Farmer MA. Predicting transition to chronic pain. Curr Opin Neurol. 2013;26(4):360-7.

30. Baliki MN, Chialvo DR, Geha PY, Levy RM, Harden RN, Parrish TB, et al. Chronic pain and the emotional brain: specific brain activity associated with spontaneous fluctuations of intensity of chronic back pain. J Neurosci. 2006;26(47):12165-73.

31. Benedetti F, Mayberg HS, Wager TD, Stohler CS, Zubieta JK. Neurobiological mechanisms of the placebo effect. J Neurosci. 2005;25(45):10390-402.

32. Wager TD, Rilling JK, Smith EE, Sokolik A, Casey KL, Davidson RJ, et al. Placebo-induced changes in FMRI in the anticipation and experience of pain. Science. 2004;303(5661):1162-7.

33. Wiech K, Kalisch R, Weiskopf N, Pleger B, Stephan KE, Dolan RJ. Anterolateral prefrontal cortex mediates the analgesic effect of expected and perceived control over pain. J Neurosci. 2006;26(44):11501-9.

34. Kringelbach ML, Rolls ET. The functional neuroanatomy of the human orbitofrontal cortex: evidence from neuroimaging and neuropsychology. Prog Neurobiol. 2004;72(5):341-72.

35. Tracey I. Getting the pain you expect: mechanisms of placebo, nocebo and reappraisal effects in humans. Nat Med. 2010;16(11):1277-83.

36. Buhle JT, Silvers JA, Wager TD, Lopez R, Onyemekwu C, Kober H, et al. Cognitive reappraisal of emotion: a meta-analysis of human neuroimaging studies. Cereb Cortex. 2014;24(11):2981-90. 


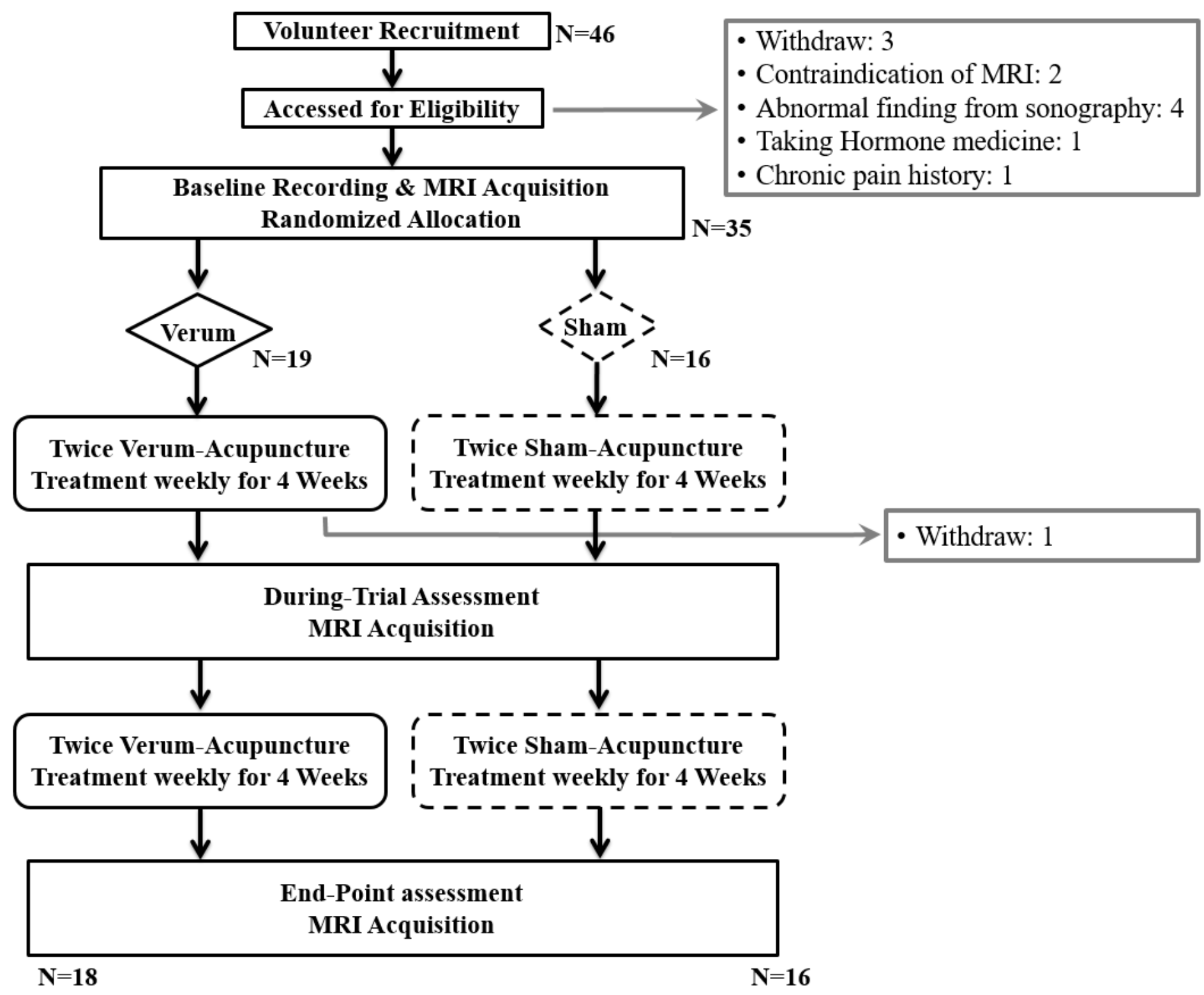

Figure 1

Flowchart of the study 


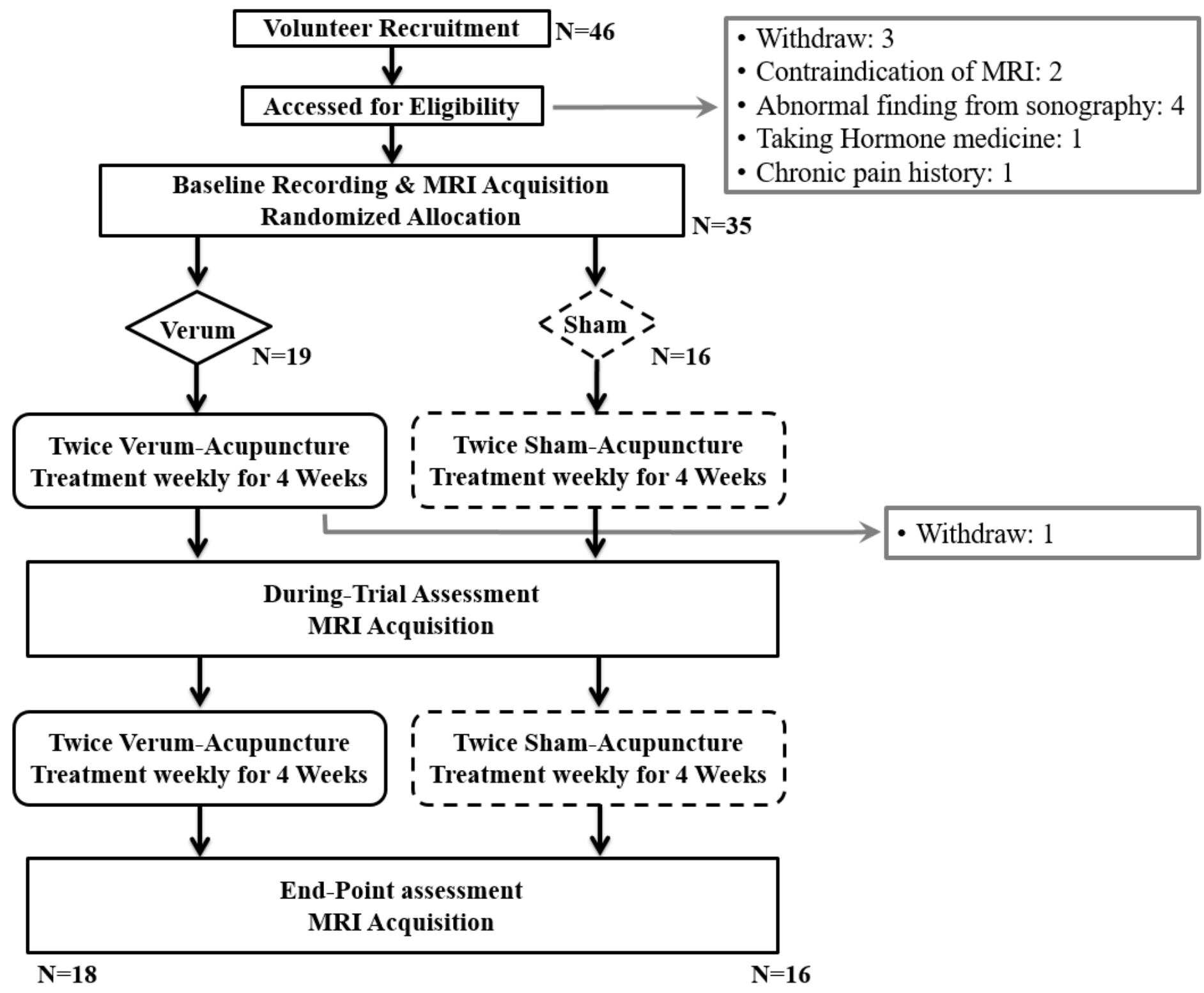

Figure 1

Flowchart of the study 


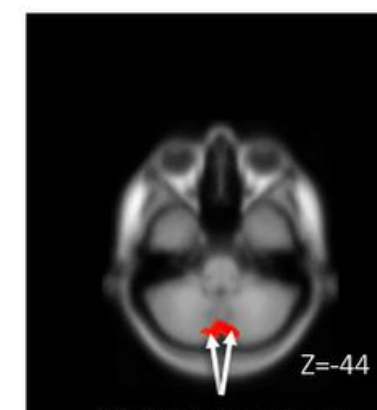

Bil Cerebellum Uvula

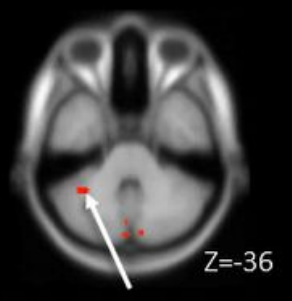

L Cerebellum Culmen

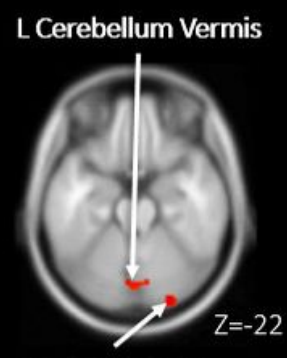

R Inf Occipital G

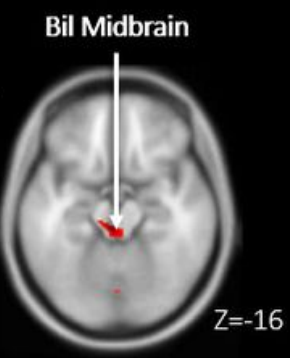

$Z=-16$

R Cuneus

\section{R Mid Frontal G}

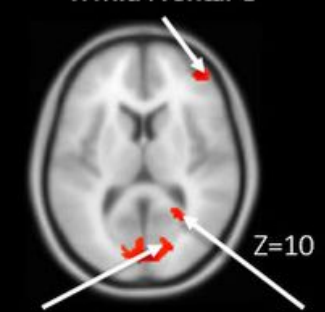

R Parahippocampal G

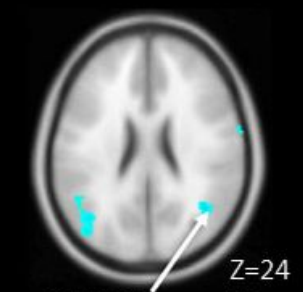

R Mid Temporal G

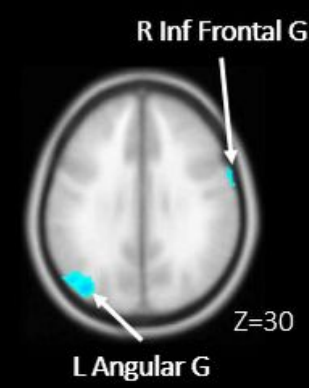

LAngular G

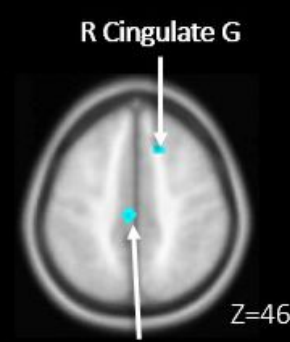

L Paracentral Lob
L Precentral G

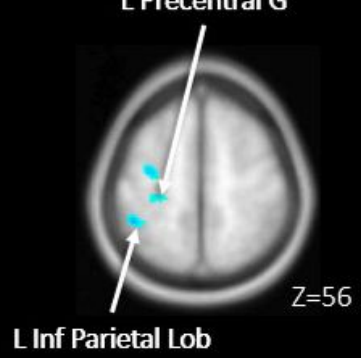

Decrease Connectivity

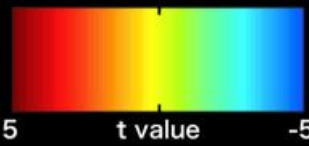

\section{Figure 2}

Changes in functional connectivity between periaqueductal gray matter and other brain regions after verum acupuncture intervention in patients with primary dysmenorrhea. After 8 weeks of verum acupuncture intervention, (upper) increased functional connectivity was found in the ventrolateral prefrontal cortex parahippocampal gyrus, visual association cortex, cerebellum, and midbrain, whereas (lower) decreased functional connectivity was found in sensorimotor regions, posterior parietal cortex and anterior cingulate cortex. Warm and cold colors denote increased and decreased functional connectivity, respectively. Abbreviation: R, right; L: left; Bil: bilateral; Mid, middle; Inf: inferior; G, gyrus; Lob: lobule. 


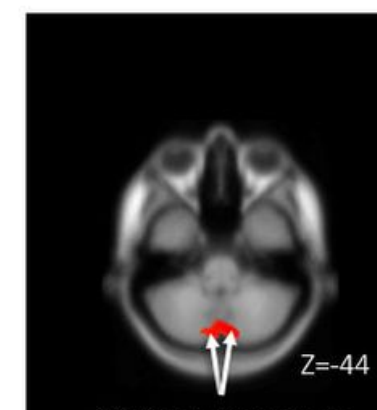

Bil Cerebellum Uvula

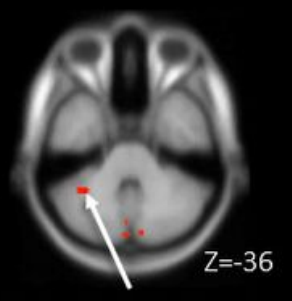

L Cerebellum Culmen

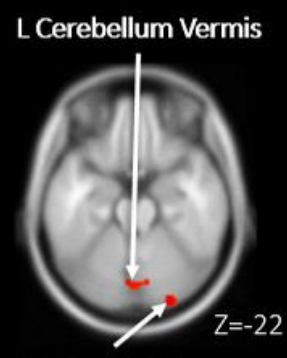

R Inf Occipital G

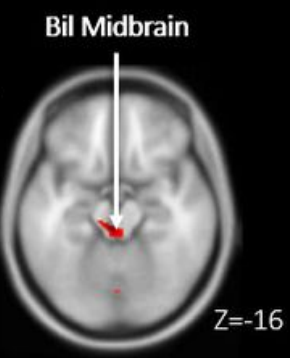

$Z=-16$

R Cuneus

\section{R Mid Frontal G}

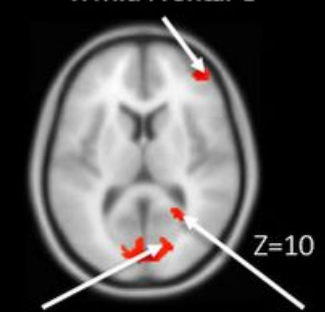

R Parahippocampal G

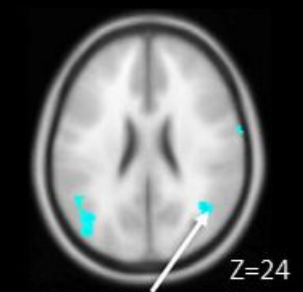

R Mid Temporal G

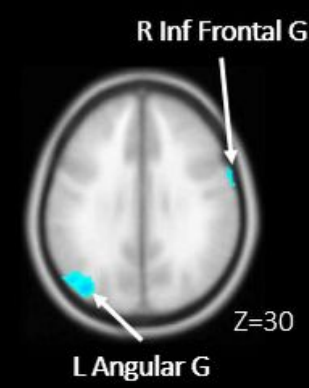

LAngular G

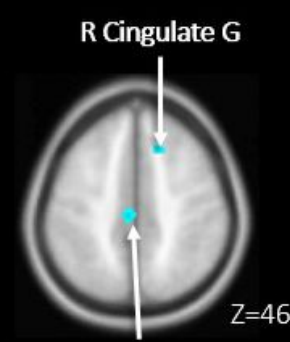

L Paracentral Lob
L Precentral G

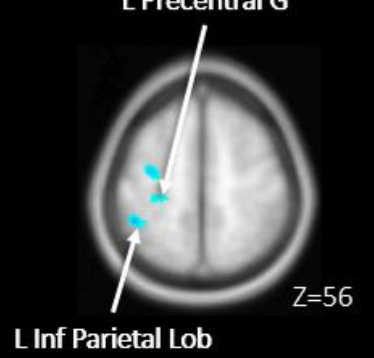

Decrease Connectivity

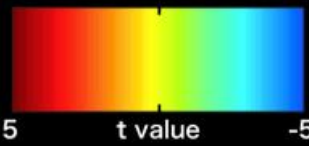

\section{Figure 2}

Changes in functional connectivity between periaqueductal gray matter and other brain regions after verum acupuncture intervention in patients with primary dysmenorrhea. After 8 weeks of verum acupuncture intervention, (upper) increased functional connectivity was found in the ventrolateral prefrontal cortex parahippocampal gyrus, visual association cortex, cerebellum, and midbrain, whereas (lower) decreased functional connectivity was found in sensorimotor regions, posterior parietal cortex and anterior cingulate cortex. Warm and cold colors denote increased and decreased functional connectivity, respectively. Abbreviation: R, right; L: left; Bil: bilateral; Mid, middle; Inf: inferior; G, gyrus; Lob: lobule. 


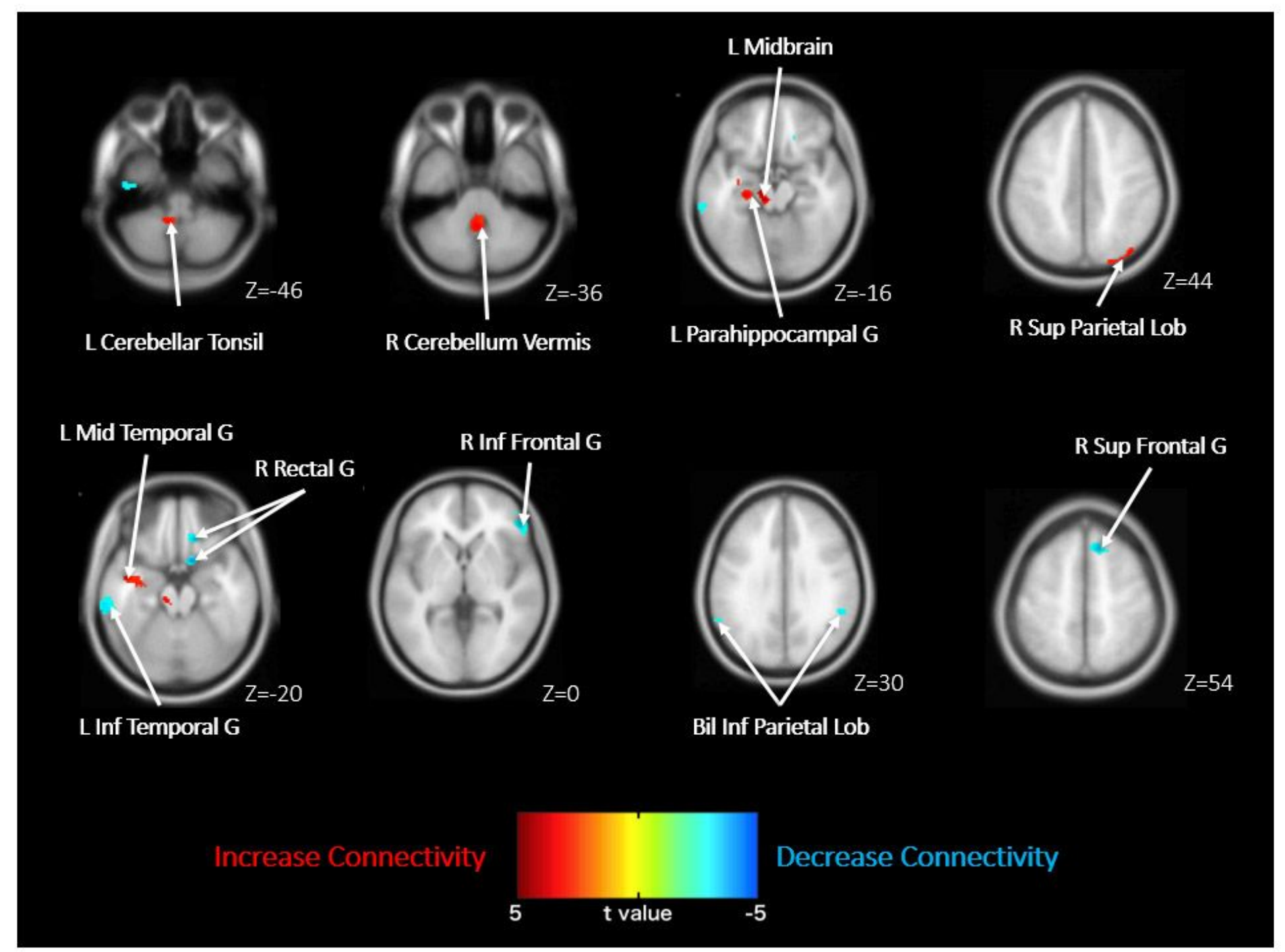

\section{Figure 3}

Changes in functional connectivity between periaqueductal gray matter and other brain regions after sham acupuncture intervention in patients with primary dysmenorrhea. After 8 weeks of sham acupuncture intervention, (upper) increased functional connectivity was found in the superior parietal lobule, middle temporal gyrus, parahippocampal gyrus, cerebellum, and midbrain, whereas (lower) decreased functional connectivity was found in the medial prefrontal cortex, ventrolateral prefrontal cortex, orbitofrontal cortex, posterior parietal cortex, and inferior temporal gyrus. Warm and cold colors denote increased and decreased functional connectivity, respectively. Abbreviation: R, right; L: left; Bil: bilateral; Sup: superior; Mid, middle; Inf: inferior; G, gyrus; Lob: lobule. 


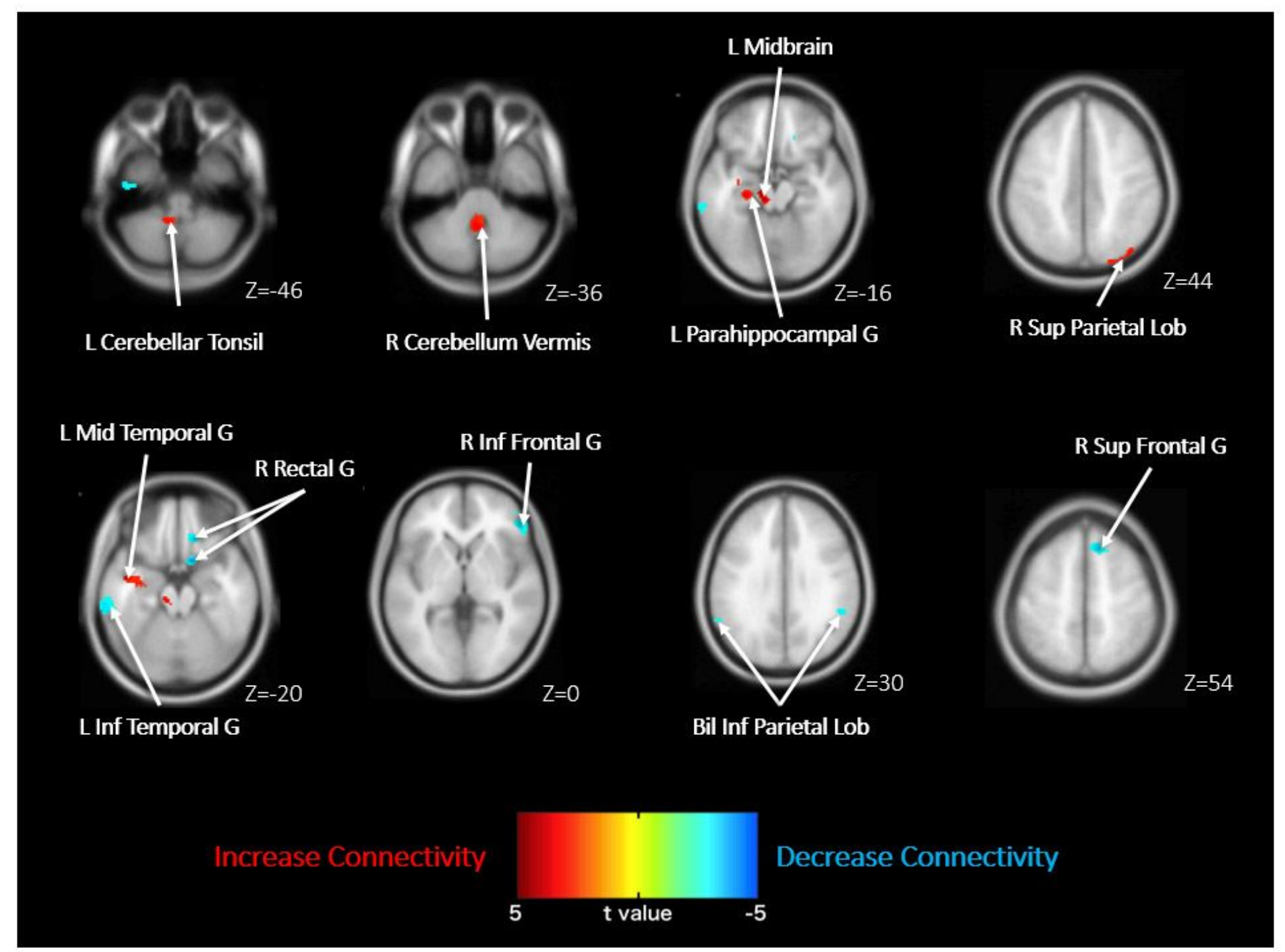

\section{Figure 3}

Changes in functional connectivity between periaqueductal gray matter and other brain regions after sham acupuncture intervention in patients with primary dysmenorrhea. After 8 weeks of sham acupuncture intervention, (upper) increased functional connectivity was found in the superior parietal lobule, middle temporal gyrus, parahippocampal gyrus, cerebellum, and midbrain, whereas (lower) decreased functional connectivity was found in the medial prefrontal cortex, ventrolateral prefrontal cortex, orbitofrontal cortex, posterior parietal cortex, and inferior temporal gyrus. Warm and cold colors denote increased and decreased functional connectivity, respectively. Abbreviation: R, right; L: left; Bil: bilateral; Sup: superior; Mid, middle; Inf: inferior; G, gyrus; Lob: lobule. 


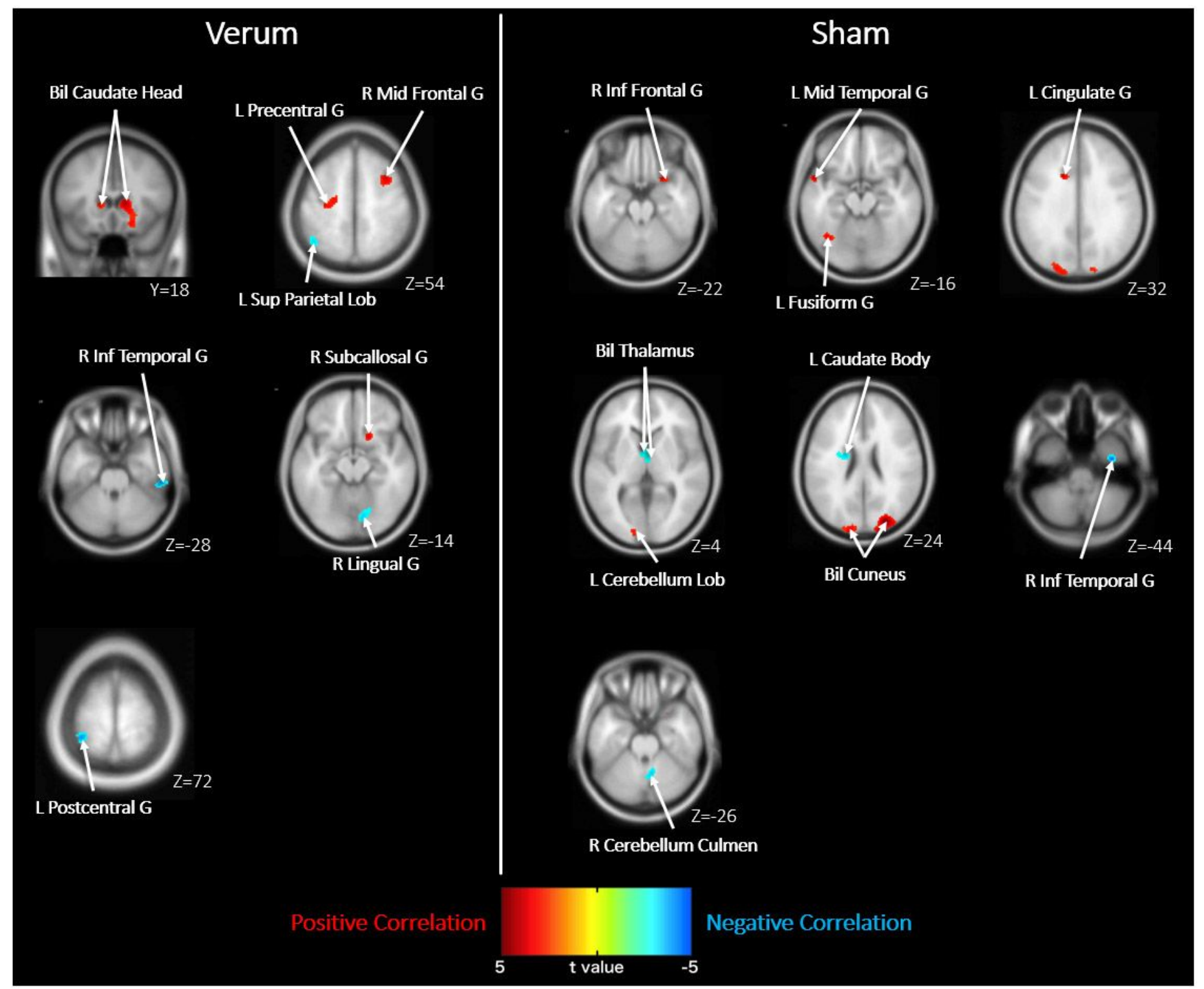

\section{Figure 4}

Correlation between the changes in functional connectivity and menstrual pain experience after acupuncture intervention in patients with primary dysmenorrhea. (Left) After 8 weeks of verum acupuncture intervention, a positive correlation between functional connectivity and menstrual pain experience was found in the motor region, orbitofrontal cortex, and caudate nucleus, whereas a negative correlation was found in the somatosensory association cortex, visual association cortex, and inferior temporal gyrus. (Right) After 8 weeks of sham acupuncture intervention, a positive correlation between functional connectivity and menstrual pain experience was found in the orbitofrontal cortex, anterior cingulate cortex, visual association cortex, basal temporal cortex, and cerebellum, whereas a negative correlation was found in the thalamus, caudate nucleus, and cerebellum. Warm and cold colors denote positive and negative correlations, respectively. Abbreviation: R, right; L: left; Bil: bilateral; Mid, middle; Inf: inferior; G, gyrus; Lob: lobule. 


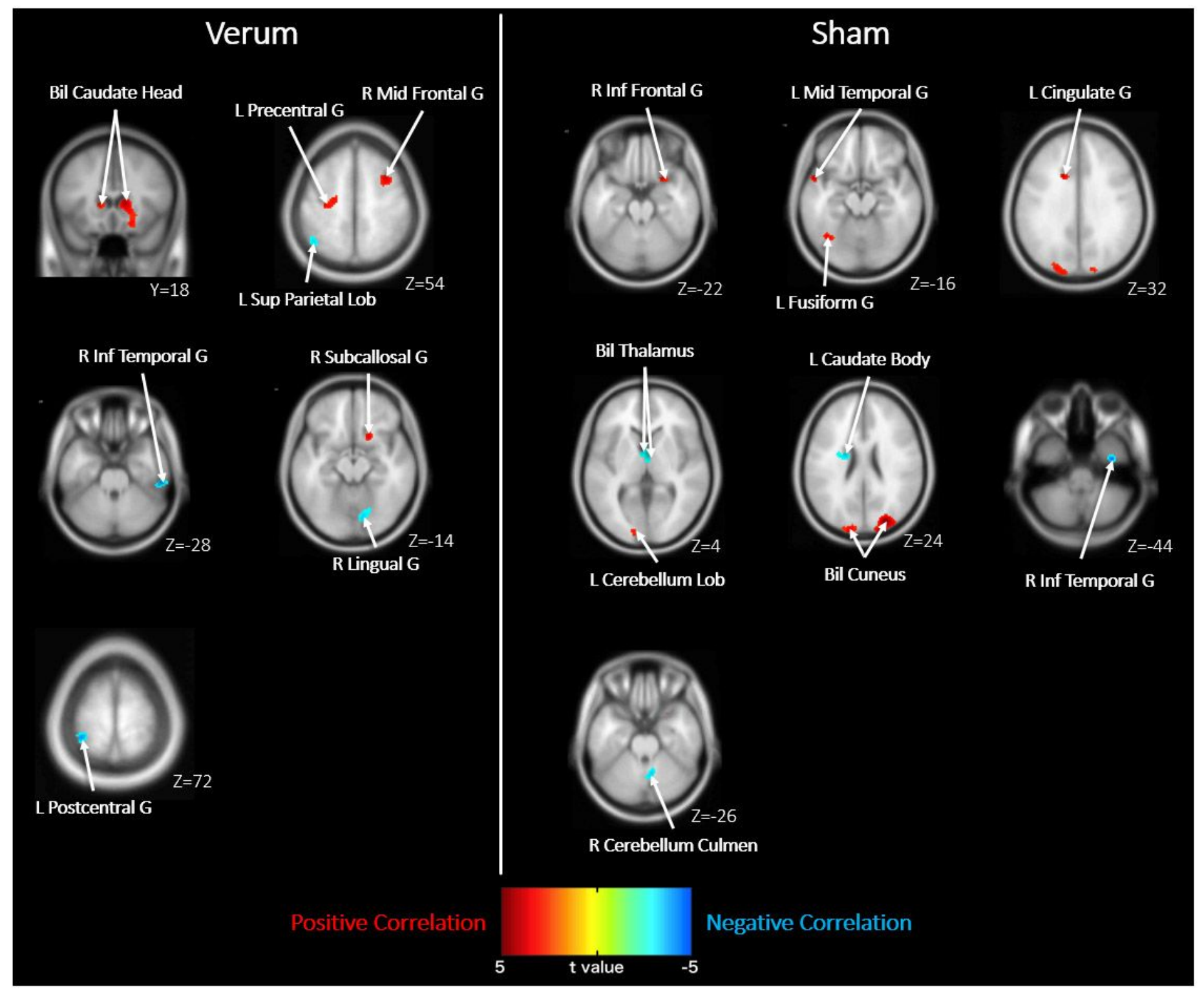

\section{Figure 4}

Correlation between the changes in functional connectivity and menstrual pain experience after acupuncture intervention in patients with primary dysmenorrhea. (Left) After 8 weeks of verum acupuncture intervention, a positive correlation between functional connectivity and menstrual pain experience was found in the motor region, orbitofrontal cortex, and caudate nucleus, whereas a negative correlation was found in the somatosensory association cortex, visual association cortex, and inferior temporal gyrus. (Right) After 8 weeks of sham acupuncture intervention, a positive correlation between functional connectivity and menstrual pain experience was found in the orbitofrontal cortex, anterior cingulate cortex, visual association cortex, basal temporal cortex, and cerebellum, whereas a negative correlation was found in the thalamus, caudate nucleus, and cerebellum. Warm and cold colors denote positive and negative correlations, respectively. Abbreviation: R, right; L: left; Bil: bilateral; Mid, middle; Inf: inferior; G, gyrus; Lob: lobule.

\section{Supplementary Files}

This is a list of supplementary files associated with this preprint. Click to download.

- Supplement.docx 
- Supplement.docx

Page 22/22 